\title{
Review Article \\ Does Task-Oriented Practice Improve Upper Extremity Motor Recovery after Stroke? A Systematic Review
}

\author{
Jackie Bosch, ${ }^{1}$ Martin J. O'Donnell, ${ }^{2}$ Susan Barreca, ${ }^{3}$ \\ Lehana Thabane, ${ }^{4}$ and Laurie Wishart ${ }^{5}$ \\ ${ }^{1}$ School of Rehabilitation Science, Population Health Research Institute, McMaster University, Room 436, \\ Institute for Applied Health Sciences 1400 Main Street West, Hamilton, ON, Canada L8S 1C7 \\ ${ }^{2}$ Population Health Research Institute, DBCVS Research Institute, McMaster University, 3rd Floor, 237 Barton St. East, \\ Hamilton, ON, Canada L8L 2X2 \\ ${ }^{3} 109$ Robinhood Drive, Dundas, ON, Canada L9H 4 G2 \\ ${ }^{4}$ Department of Clinical Epidemiology and Biostatistics, Centre for Evaluation of Medicines, McMaster University, \\ 105 Main St. East, Level PI, Hamilton, ON, Canada L8N 1 G6 \\ ${ }^{5}$ School of Rehabilitation, Faculty of Health Sciences, McMaster University, 1400 Main Street West, Hamilton, ON, Canada L8S 1C7
}

Correspondence should be addressed to Jackie Bosch; Jackie.bosch@phri.ca

Received 4 November 2013; Accepted 12 December 2013; Published 18 February 2014

Academic Editors: H. McNaughton and A. Slivka

Copyright (C) 2014 Jackie Bosch et al. This is an open access article distributed under the Creative Commons Attribution License, which permits unrestricted use, distribution, and reproduction in any medium, provided the original work is properly cited.

Background. Stroke commonly affects upper extremity motor abilities, yet there has been very limited success in developing effective rehabilitation interventions to remediate motor impairments, particularly for the upper extremity. Objective. To determine if taskoriented practice administered soon after stroke is more effective than usual care in improving poststroke upper extremity motor recovery and to explore the optimal amount of practice. Methods. A systematic review of the literature was performed from 1950 to November 2012, to identify randomized controlled trials of task-oriented practice compared to usual care, or to different amounts of task-oriented practice to improve motor impairment and activity. Studies were excluded if specific types of interventions were used as comparators or if they were of poor methodological quality. Results. Six studies met the review criteria. Three of the six studies demonstrated a statistically significant effect of task-oriented practice. Study results could not be pooled because of a lack of homogeneity in populations and intervention. Conclusions. The results demonstrate that an increase in the amount of task-oriented practice after stroke may result in less upper extremity impairment; further research on both effect and required dosage is needed as results are inconsistent.

\section{Introduction}

Stroke is the fifth leading cause of disability internationally [1] and it is likely that this is an underestimate of the absolute level of functioning that is lost, especially in lowincome countries [2]. Stroke results in disability through loss of function from motor, cognitive, or sensory impairments either individually or in combination. Although stroke survivors can compensate for some of these losses, they often experience substantial residual functional deficits.

Motor impairment is the most common deficit after stroke and the major contributor to functional limitations [3]. Motor impairments are a principal target of rehabilitation interventions, and many novel therapeutic approaches for motor recovery have emerged in recent decades [3-18]. Research has focused on lower limb motor impairment more than upper limb partly because lower limb interventions are more easily described, outcomes are more easily quantified, and mobility is considered a key functional consideration after stroke. However, among those who have had a stroke, upper limb recovery is also considered important because it is integral to independence in many activities of daily living [19]. Therefore, interventions designed and tested specifically for upper extremity motor recovery are required.

Motor recovery interventions have been developed based on a variety of theoretical paradigms. Interventions may look 
similar; however, the expected mechanism of effect differs. Rigorous evaluation of novel interventions has been a major challenge due to the complexity of the interventions [20] and the variability of the poststroke populations (e.g., acute, subacute, and chronic). Furthermore, the methodological limitations of completed trials (e.g., small sample sizes) have resulted in studies that are underpowered to detect the modest effects expected from rehabilitation interventions [21]. In situations where there are numerous underpowered clinical trials, meta-analysis may prove useful in estimating a potential treatment effect. However, conducting and interpreting meta-analyses of clinical trials evaluating interventions for upper limb recovery has been challenging because of the heterogeneity of interventions and outcomes. This has made interpretation of the evidence difficult for guideline committees as well as clinicians trying to determine "best practice."

One promising upper extremity motor recovery intervention is task-oriented practice $[3,6,22,23]$. While the role of task practice in improving general motor performance has long been recognized as important [24], the evidence to support its effect on poststroke motor recovery is based on small clinical trials and observational studies. A metaanalysis of clinical trials evaluating task-oriented practice has reported modest benefits in functional outcomes for lower limb motor, but not for upper limb impairments [6]. The studies included in the meta-analysis were small, heterogeneous in terms of population and outcome, and some used active comparators that may have also used task practice. Therefore, it is not surprising that an effect on upper extremity impairment was not evident.

Determining whether task-oriented practice is effective depends in part at least, on understanding the "amount" of practice necessary to improve motor performance, or as it has been called by some, the "dosage" of task-oriented practice. Dosage is a commonly used term when prescribing pharmaceuticals, and it describes the concentration of the active ingredient as well as the frequency and duration of administration. Although the application of this concept to rehabilitation therapies was first proposed over 15 years ago [25], implementation in clinical and research settings has been slow, potentially because of a lack of consensus regarding the definition [26]. Most rehabilitation clinicians and researchers agree that the frequency and duration of an intervention can be quantified using minutes per day and number of days over which an intervention is administered. There is less agreement about the definition of intensity, with some considering the more physiologic interpretation (e.g., the energy expenditure, which can be measured by scales such as Metabolic Equivalent of Task (MET)) [27, 28] while others define it as the minutes per day on a prescribed protocol, where the goal is the maximum amount of task practice in the time provided $[29,30]$. Given that intensity describes the concentration of the active ingredient in task-oriented practice, time on task, as opposed to the energy expended on task, is the more appropriate measure. The argument is however somewhat academic, as studies generally do not measure intensity. In their systematic review, Langhorne et al. [3] noted that high-intensity rehabilitation may be beneficial; however, the available data did not allow for quantification of the level of intensity, a conclusion that is consistent with other studies and reviews $[6,13,28,31,32]$. Therefore, although there is recognition of the importance of understanding the elements of dosage, studies to date have not been able to clearly determine the parameters, and in particular to quantify intensity. This limitation, along with the methodological limitations of many studies, may lead to inaccurate conclusions, such as those reported in a recent review, that higher intensity rehabilitation does not improve functional recovery [33].

Studies providing upper extremity task-oriented practice, given during the subacute phase (prior to the development of long-term compensatory strategies), in an inpatient setting (most likely the highest dosage) where the comparator group is usual practice (as opposed to other interventions that have not proven effective), would provide the most accurate estimate of the effect of task-oriented practice on upper extremity motor recovery in the first months after stroke and may allow for quantification of a threshold dose for motor recovery. Therefore, we performed a systematic review (a) to determine if task-oriented practice, administered early after stroke, is more effective than usual care in improving poststroke upper extremity motor recovery and (b) to explore whether there is a "dose" response within clinical trials in terms of duration, frequency and intensity.

\section{Methods}

2.1. Inclusion/Exclusion Criteria. Studies considered for inclusion in this systematic review met the following criteria:

(a) randomized participants (i.e., randomized controlled trials (RCTs)), as these studies provide the most valid estimate of effectiveness;

(b) included an adult population with upper extremity motor deficits, three months or less after stroke (with or without lower extremity deficits);

(c) used upper extremity task practice as the intervention, with enough information to quantify the amount of upper extremity task practice (not necessarily the intensity, but at least frequency and duration);

(d) used at least one outcome measure that quantified upper extremity motor recovery (either at the International Classification of Functioning and Health level of Body Function or Activities and Participation [34]);

(e) published in full in English.

Studies were excluded from the review if they met the following criteria:

(a) used any specific intervention method (e.g., NeuroDevelopmental Therapy, Motor Relearning Program, Constraint Induced Movement Therapy, exercise, Virtual Reality, Mental Imagery, Therapeutic Electrical Stimulation, and Transcranial Magnetic Stimulation), for either the intervention or control 
groups. Studies comparing specific interventions were excluded because it is difficult to quantify the amount of task performance and task is not necessarily the focus of the intervention or the "active ingredient";

(b) were of poor methodological quality that is, a score of $<4$ using the Centre for Evidence Based Medicine, Randomized Controlled Trial Critical Appraisal sheet (http://www.cebm.net/index.aspx?o=1157) which assessing internal validity. This scale is similar to other scales of clinical trial quality, but focuses specifically on issues of internal validity that are key to obtaining valid results.

2.2. Information Sources, Search, and Study Collection. The search for studies was done in multiple phases. Given the lack of consistency in the use of both subject headings and keywords, an initial search was performed using the following terms in various combinations; "stroke," "motor learning," "task," "arm," and similar terms (e.g., cerebrovascular accident, motor recovery, practice) in the following databases: CINAHL (EBSCO Host, 1981-November 2012), MEDLINE R (Ovid) (1950-November 2012), EMBASE (OvidSP, 1980November 2012). Two reviewers (Jackie Bosch (JB), Susan Barreca (SB)) independently reviewed the list of titles and chose articles for abstract review based on the inclusion criteria provided above. The same two researchers (JB, SB) independently reviewed the abstracts and if selected by either reviewer the articles went for complete text review. Full articles were then reviewed against the inclusion and exclusion criteria, and the methodological quality of the studies was appraised using the Centre for Evidence Based Medicine Randomized Controlled Trial Critical Appraisal sheet (http://www.cebm.net/index.aspx?o=1157). Agreement rates for study inclusion were $85 \%$, and a consensus approach was used when there was discrepancy.

A final review of the remaining articles was performed by Laurie Wishart (LW) and Martin O'Donnell (MO) to confirm appropriateness for inclusion.

2.3. Data Collection Process, Data Items, and Risk of Bias in Individual Studies. Data were extracted from studies by JB and independently reviewed by LW and MO. Data included mean age, inclusion criteria for upper extremity movement, time since stroke, amount of practice in each group (minutes per day, days per weeks, and number of weeks), upper extremity motor recovery outcome measures (either at the Body Structure or Activities and Participation level, based on the International Classification of Functioning Disability and Health by the World Health Organization [35]), and timing of the assessment of the primary outcome measure. If the authors did not indicate which outcome was primary, measures were chosen based on the expected sensitivity to the intervention and administration time in closest proximity to the end of the intervention period, as this is when the largest effect is expected to occur, opposed to weeks or months thereafter. All data were extracted from publications.
2.4. Risk of Bias within and across Studies. Using the Centre for Evidence Based Medicine Randomized Controlled Trial Critical Appraisal sheet (http://www.cebm.net/index .aspx? $\mathrm{o}=1157$, internal validity section), one point was given for a "yes" to each question, with a maximum score of 6 for each study. Because of the nature of the interventions, participants were not blinded to their treatment group in any study; however, outcome assessment was often completed by assessors who were blinded to treatment allocation and in such circumstances, one point was given for blinded outcome assessment.

In terms of potential for bias across the studies, there is always a risk of publication bias; that is, studies that are positive are more likely to get published. Half of the studies in this review demonstrated a statistically significant effect (3 out of 6).

2.5. Data Synthesis. The reporting of the results of this review is done in accordance with the PRISMA guideline [36]. Studies meeting the inclusion criteria were heterogeneous in study population (i.e., amount of upper extremity motor deficit) and interventions evaluated (i.e., different taskoriented approaches); and such as, the authors concluded that meta-analysis would not be appropriate. Therefore, the results are reported descriptively with some narrative summaries.

\section{Results}

3.1. Study Selection. The initial search resulted in a list of 309 titles, of which 60 were excluded and 299 abstracts were reviewed. A hand search of bibliographies resulted in an additional 62 citations for review. A total of 361 abstracts were reviewed of which 136 (38\%) were found eligible for full text review. From the full review, 23 (17\%) studies were eligible for inclusion and underwent a third review by a second set of reviewers, who excluded an additional 17 articles. The remaining 6 articles represent 6 unique studies that were included in the review (see Figure 1).

The three most common reasons for excluding articles/studies were (a) use of a specific intervention as a comparator (38\%), (b) the article was a review (critical or systematic) $(23 \%)$, and (c) the study design was not a randomized controlled trial or practice was not used as an intervention (20\%).

3.2. Study Characteristics. The key characteristics of the six studies included in this review are provided in Table 1 . The mean age for the participants' varied from 50 to 75 years. All studies required participants to have motor impairment in the affected upper extremity and two also required that participants had some upper extremity movement. Although tools used to measure severity of deficits at baseline varied across studies, results indicated moderate upper extremity impairment in each study. Participants were recruited within about six weeks of stroke onset. Ethics approval from the local institution was obtained for each study and informed consent was obtained from each study participant. 


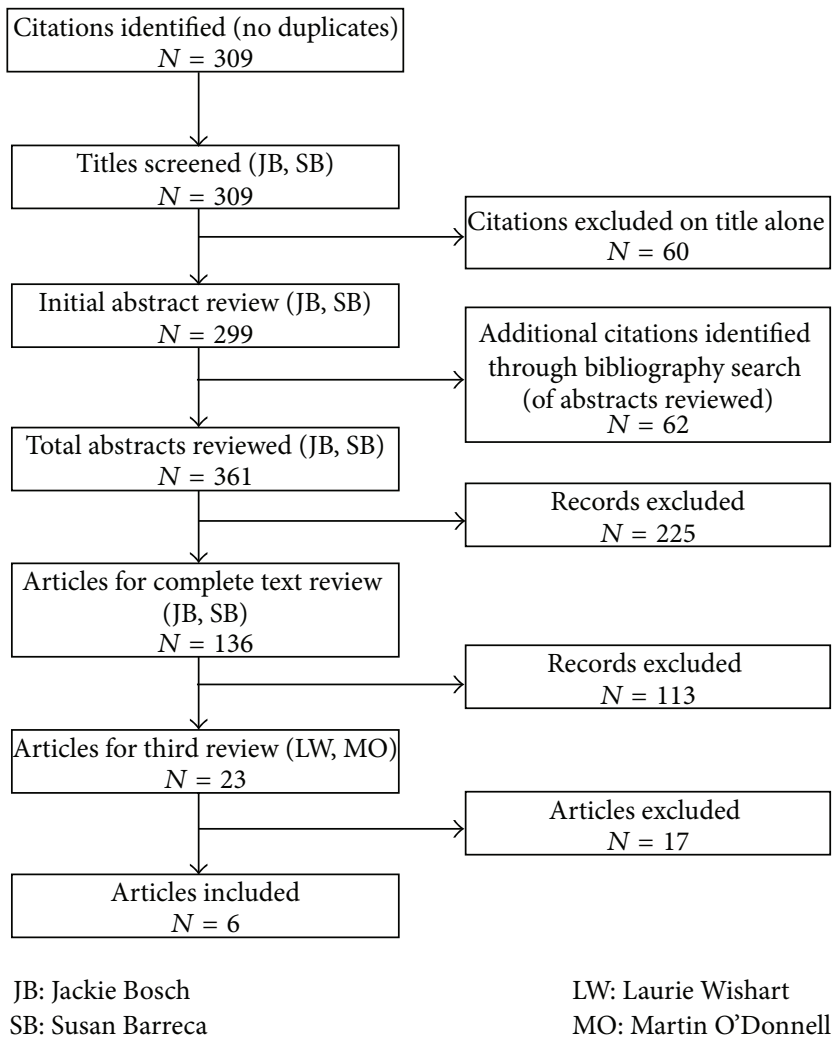

Figure 1: Flow diagram of the literature search.

All studies provided a brief explanation of the experimental (E) and control (C) interventions, with functional task practice and repetition as key elements of the experimental intervention. Strength training was also included in the experimental intervention for 5 of the 6 studies [37-41]. Additional details on the experimental intervention protocolwere publically available for one study [40] (http://www .neostrokenetwork.com/newportal/Portals/0/Education\%20 Documents/videoconf\%20handout/GRASP_Manual11492 .pdf), while two authors indicated that protocols were developed but were not publically available [41, 42]. One study formally evaluated different durations of the same intervention [39]. In this three arm study, the same intervention was given for 1,2 and 3 hours per day to each of the groups, 5 days per week for six weeks.

The most commonly used outcome assessment was the Action Research Arm Test (ARAT) which uses usual activities and movements to assess grasp, grip, pinch, and overall upper extremity movement. It was the primary outcome measure in three of the studies $[38,39,41]$ and a secondary outcome measure in another [40], and was therefore the outcome measure reported for each of these studies, to allow for comparison between studies. The remaining two studies used measures of upper extremity motor recovery after stroke as the primary outcome, and data for each of these measures were provided [37, 42].

All studies included in this review received a score of 4 or higher using the Centre for Evidence Based Medicine Randomized Controlled Trial Critical Appraisal sheet (see, Table 2). The most common limitations in the studies were lack of complete follow up of participants at study end (five studies), followed by an imbalance of key prognostic variables between groups at baseline (three studies).

3.3. Study Outcomes. Table 3 provides both the baseline score and end of intervention score for each of the studies. In three of the six studies, statistically significant between group differences in the ARAT at study end were demonstrated in favour of additional, deliberate, task-oriented practice $(P \leq$ 0.01 ) [39-41]. Two other studies suggested an improvement in the task-oriented practice group (versus control); however, this was not statistically significant $[38,42]$. One study did not show any significant difference [37].

The issue of the effect of differing amounts of therapy was addressed by one study, where a dose response effect was suggested with higher dosages of task-oriented practice resulting in improved activity, although a statistically significant difference was evident only between the lowest and highest dose group [39].

\section{Discussion}

4.1. Main Findings. Based on the six studies identified in this systematic review, there is some evidence of a potentially beneficial effect of task-oriented practice in upper extremity motor recovery after stroke. Results of the study by Han et al. [39] indicate there may be a dose response relationship, 
TABLE 1: Characteristics of Included Studies.

\begin{tabular}{|c|c|c|c|c|}
\hline First author Year & $\begin{array}{l}n \text { participants } \\
\text { Description }\end{array}$ & $\begin{array}{l}\text { Description of intervention } \\
\text { Experimental (E), Control (C) }\end{array}$ & $\begin{array}{l}\text { Additional Deliberate } \\
\mathrm{u} / \mathrm{e} \text { Practice }\end{array}$ & $\begin{array}{l}\mathrm{u} / \mathrm{e} \text { or Functional Outcome } \\
\text { Measures }\end{array}$ \\
\hline hassett, & $\begin{array}{l}n=30,2 \text { groups } \\
55 \text { yrs (mean) } \\
\text { No u/e entry criteria } \\
43 \text { days after stroke } \\
\text { (avg.) }\end{array}$ & $\begin{array}{l}\text { In addition to usual care; } \\
\text { E: upper limb group, functional } \\
\text { tasks to improve reach, grasp, } \\
\text { coordination, } n=15 \\
\text { C: mobility group, sit-to-stand, } \\
\text { walking, stairs, } n=15\end{array}$ & $\begin{array}{l}\text { A: upper limb group: } 5 \\
\text { hours per week for } 4 \text { weeks } \\
\text { C: mobility group: no } \\
\text { additional upper extremity } \\
\text { practice }\end{array}$ & $\begin{array}{l}\text { Motor assessment scale } \\
\text { (MAS) upper } \\
\text { limb/extremity } \\
\text { MAS hand } \\
\text { Jebsen hand function test (3 } \\
\text { subsets) }\end{array}$ \\
\hline $\begin{array}{l}\text { Donaldson, } \\
2009 \text { [38] }\end{array}$ & $\begin{array}{l}n=30,3 \text { groups } \\
72.8 \text { yrs (mean) } \\
\text { Some movement } \\
\text { required in paretic u/e } \\
20 \text { days after stroke } \\
\text { (avg.) }\end{array}$ & $\begin{array}{l}\text { In addition to usual care; } \\
\text { E: Functional upper extremity } \\
\text { training, using progressive task } \\
\text { training, } n=10 \\
\mathrm{C} 1 \text { : additional session of usual } \\
\text { (exercise based) care, } n=10 \\
\mathrm{C} 2 \text { : nothing in addition to usual } \\
\text { (exercise based) care, } n=10\end{array}$ & $\begin{array}{l}\text { A: } 4 \text { hours per week for six } \\
\text { weeks } \\
\text { C1: } 4 \text { hours per week for six } \\
\text { weeks } \\
\text { C2: no additional upper } \\
\text { extremity practice }\end{array}$ & $\begin{array}{l}\text { Action Research Arm Test } \\
(\text { ARAT })^{\wedge} \\
\text { Nine hole peg test (9HPT) } \\
\text { Grip force } \\
\text { Pinch force } \\
\text { Elbow flexion/extension } \\
\text { force }\end{array}$ \\
\hline
\end{tabular}

In addition to usual care, strength training and functional activity practice in three doses: $n=32,3$ groups 50.2 yrs (mean)

Han, 2013 [39] u/e impairment, no spasticity, no pain 40 days after stroke (avg.)
E: 3 hours/day, 5 days/week, $n=11$

C1: 2 hours/day, 5 days/week, $n=10$

C2: 1 hour/day, 5 days/week, $n=11$
A: 15 hours/week for 6

weeks

C1: 10 hours/week for 6 weeks

C2: 5 hours/week for 6 weeks
ARAT $^{\wedge}$

Fugl-Meyer (arm items) Barthel index $n=103,2$ groups

69 yrs (mean)

Harris, 2009

[40]

mov't

20 days after stroke

(avg.)
In addition to usual care:

E: task-oriented, unsupervised

home-based exercise program

C: education program

\section{A: 3.75 hours/week for 4}

weeks

C: 0.75 hours/week for 4 weeks
Chedoke Arm and Hand

Activity Inventory

$(\text { CAHAI })^{\wedge}$

ARAT

Motor activity Log-14

(MAL)

Grip strength

Short form-12 (SF-12)

Pain visual analog scale

Fatigue severity scale
In addition to usual care:

$\mathrm{E}$ : intense arm training,

$\mathrm{C} 1$ : intense leg training, sitting/standing/walking activities, $n=31$

C2: Control (air splint), $n=37$ functional exercises, $n=33$

\begin{abstract}
A: 2.5 hours per week, 20
weeks

C1: No additional upper extremity practice C2: No additional upper extremity practice
\end{abstract}

ARAT $^{\wedge}$

Barthel Index

Nottingham Health Profile

Sickness Impact Profile

Frenchay Activities Index

$n=64,3$ groups
Mean age, $95 \%$ between
35 and 75 years
Upper extremity
impairment (inferred
from FIM score)
16 days after stroke
(avg.)

In addition to usual care:

E: functional task practice (FTP) (repetitive and progressive), $n=22$

$\mathrm{C} 1$ : strength and motor control training (ST), exercise based, $n=21$

C2: usual care (UC), $n=21$
A: 5 hours per week for 4 weeks (task) C1: 5 hours per week for 4 weeks (exercise) C2: no additional task-oriented practice
Functional test of the hemiparetic upper extremity (FTHUE) ${ }^{\wedge}$ FIM Fugl-Meyer Upper extremity (motor, sensory, ROM) Grip force

Pinch force Shoulder/elbow/wrist torque FIM self-care 
TABLE 2: Assessment of the risk of Bias.

\begin{tabular}{|c|c|c|c|c|c|c|c|}
\hline First Author, year & Randomized & $\begin{array}{l}\text { Similarity of } \\
\text { groups }\end{array}$ & $\begin{array}{l}\text { Groups } \\
\text { treated } \\
\text { equally }\end{array}$ & $\begin{array}{c}\text { All } \\
\text { participants } \\
\text { accounted for }\end{array}$ & $\begin{array}{l}\text { Analysed in } \\
\text { group } \\
\text { assigned }\end{array}$ & $\begin{array}{c}\text { Outcome } \\
\text { measure } \\
\text { blinded }\end{array}$ & Score $(/ 6)$ \\
\hline Blennerhassett, 2004 [37] & $\mathrm{Y}$ & $\mathrm{N}$ & $\mathrm{Y}$ & $\mathrm{Y}$ & $\mathrm{Y}$ & $\mathrm{Y}$ & 5 \\
\hline Donaldson, 2009 [38] & $\mathrm{Y}$ & $\mathrm{N}$ & $\mathrm{Y}$ & $\mathrm{N}$ & $\mathrm{Y}$ & $\mathrm{Y}$ & 4 \\
\hline Han, 2013 [39] & $\mathrm{Y}$ & $\mathrm{N}$ & $\mathrm{Y}$ & $\mathrm{N}$ & $\mathrm{Y}$ & $\mathrm{Y}$ & 4 \\
\hline Harris, 2009 [40] & $\mathrm{Y}$ & $\mathrm{Y}$ & $\mathrm{Y}$ & $\mathrm{Y}$ & $\mathrm{Y}$ & $\mathrm{Y}$ & 6 \\
\hline Kwakkel, 1999 [41] & $\mathrm{Y}$ & $\mathrm{Y}$ & $\mathrm{Y}$ & $\mathrm{N}$ & $\mathrm{Y}$ & $\mathrm{Y}$ & 5 \\
\hline Winstein, 2004 [42] & $\mathrm{Y}$ & $\mathrm{Y}$ & $\mathrm{Y}$ & $\mathrm{N}$ & $\mathrm{Y}$ & $\mathrm{N}$ & 4 \\
\hline
\end{tabular}

Y: yes; N: no; total score $=$ sum of Y's.

TABLE 3: Results for primary outcome measure.

\begin{tabular}{|c|c|c|c|c|c|c|c|}
\hline $\begin{array}{l}\text { First author, } \\
\text { year }\end{array}$ & $\begin{array}{l}\text { Primary } \\
\text { outcome }\end{array}$ & $\begin{array}{c}\text { Timing of } \\
\text { measurement }\end{array}$ & Statistic & & \multicolumn{2}{|c|}{ Results: experimental (E) and control (C) } & $P$ \\
\hline \multirow[t]{3}{*}{$\begin{array}{l}\text { Blennerhassett, } \\
2004 \text { [37] }\end{array}$} & & & & \multicolumn{3}{|c|}{$\begin{array}{c}\text { E: } 5 \text { hours/wk C: no addl. deliberate u/e practice } \\
\quad n=15 \quad n=15\end{array}$} & \\
\hline & \multirow{2}{*}{$\begin{array}{l}\text { MAS upper } \\
\text { limb/extremity }\end{array}$} & Baseline & $\begin{array}{l}\text { Median } \\
(Q 1-Q 3)\end{array}$ & $5(2-5)$ & \multicolumn{2}{|c|}{$5(1-6)$} & NR \\
\hline & & $\begin{array}{l}4 \text { weeks after rand } \\
\text { (end of intervention) }\end{array}$ & $\begin{array}{l}\text { Median } \\
(Q 1-Q 3) \\
\end{array}$ & $6(5-6)$ & \multicolumn{2}{|c|}{$6(4-6)$} & NR \\
\hline \multirow[t]{3}{*}{$\begin{array}{l}\text { Donaldson, } \\
2009[38]\end{array}$} & & & & $\begin{array}{c}\text { E: } 4 \text { hours/wk } \\
\text { task } \\
n=10^{*} \\
\end{array}$ & $\begin{array}{c}\text { C1: } 4 \text { hours/wk } \\
\text { exercise } \\
n=10 \\
\end{array}$ & $\begin{array}{c}\text { C2: no addl. u/e } \\
\text { practice } \\
n=8\end{array}$ & \\
\hline & \multirow[b]{2}{*}{ ARAT $^{\wedge}$} & Baseline & $\begin{array}{l}\text { Median } \\
Q 1-Q 3\end{array}$ & $27.0(11.0)$ & $34.5(26.0)$ & $28.0(17.0)$ & \\
\hline & & $\begin{array}{l}6 \text { weeks after rand } \\
\text { (end of intervention) }\end{array}$ & $\begin{array}{l}\text { Median } \\
\text { (Q1-Q3 } \\
\text { change) }\end{array}$ & $19.50(22.00)$ & $8.00(13.25)$ & $11.50(21.00)$ & 0.232 \\
\hline \multirow[t]{3}{*}{ Han, 2013 [39] } & & & & $\begin{array}{c}\text { E: } 15 \\
\text { hrs/week } n= \\
10\end{array}$ & $\begin{array}{c}\text { C1: } 10 \text { hours/week } \\
n=10\end{array}$ & $\begin{array}{c}\text { C2: } 5 \text { hours/week } \\
n=10\end{array}$ & \\
\hline & \multirow[b]{2}{*}{ ARAT } & \multirow{2}{*}{$\begin{array}{c}\text { Baseline } \\
6 \text { weeks after rand } \\
\text { (end of intervention) }\end{array}$} & Mean (SD) & $1.10(1.52)$ & $1.50(1.58)$ & $0.80(1.14)$ & 0.386 \\
\hline & & & Mean (SD) & $10.90(3.60)$ & $8.70(4.62)$ & $5.30(3.40)$ & 0.008 \\
\hline \multirow[t]{3}{*}{$\begin{array}{l}\text { Harris, } 2009 \\
{[40]}\end{array}$} & & & & $\begin{array}{c}\text { E: } 3.75 \\
\text { hours/week } \\
n=50 \\
\end{array}$ & $\begin{aligned} \text { C: } 0.75 \mathrm{l} \\
\quad n=\end{aligned}$ & $\begin{array}{l}\text { hours/week } \\
=53\end{array}$ & \\
\hline & \multirow{2}{*}{ ARAT } & Baseline & Mean & 31.1 & & 31.0 & \\
\hline & & $\begin{array}{l}4 \text { weeks after rand } \\
\text { (end of intervention) }\end{array}$ & Mean (SD) & $42.8(9.20)$ & 38.0 & $(9.47)$ & 0.025 \\
\hline \multirow[t]{3}{*}{$\begin{array}{l}\text { Kwakkel, } 1999 \\
{[41]}\end{array}$} & & & & $\begin{array}{c}\text { E: } 2.5 \\
\text { hours/week } \\
n=29\end{array}$ & $\begin{array}{c}\text { C1: no addl. U/E } \\
n=26\end{array}$ & $\begin{array}{c}\text { C2: no addl. u/e } \\
\text { extremity } \\
n=34\end{array}$ & - \\
\hline & \multirow{2}{*}{ ARAT } & Baseline & $\begin{array}{l}\text { Median } \\
(Q 1-Q 3)\end{array}$ & $0(0-1)$ & $0(0-6)$ & $0(0-0)$ & - \\
\hline & & $\begin{array}{l}20 \text { weeks after rand } \\
\text { (end of intervention) }\end{array}$ & $\begin{array}{l}\text { Median } \\
(Q 1-Q 3)\end{array}$ & $9(0-39)$ & $2(0-56)$ & $0(0-2)$ & 0.01 \\
\hline \multirow[t]{3}{*}{$\begin{array}{l}\text { Winstein, } 2004 \\
\text { [42] }\end{array}$} & & & & $\begin{array}{c}\text { E: } 5 \\
\text { hours/week } \\
\text { task } \\
n=20\end{array}$ & $\begin{array}{c}\text { C1: } 5 \text { hours/week } \\
\text { exercise } \\
n=20\end{array}$ & $\begin{array}{c}\text { C2: no addl. u/e } \\
\text { practice } \\
n=20\end{array}$ & \\
\hline & \multirow[b]{2}{*}{ FTHUE } & \multirow{2}{*}{$\begin{array}{c}\text { Baseline } \\
4 \text { weeks after rand } \\
\text { (end of intervention) }\end{array}$} & Mean (SD) & $4.30(5.35)$ & $5.15(5.97)$ & $5.40(4.30)$ & 0.83 \\
\hline & & & Mean (SD) & $4.70(4.27)$ & $4.25(4.33)$ & $3.35(3.63)$ & 0.61 \\
\hline
\end{tabular}

${ }^{*}$ Note that Ns provided in this section may differ from Table 1 as these are the numbers used for analysis; ${ }^{\wedge}$ ARAT: higher score $=$ better outcome; $n$ : sample size. MAS upper limb/extremity: motor assessment scale, upper limb/extremity section (score range of $0-18$, high score indicating normal ability). Q1: 1st quartile; Q3: 3rd quartile; NR: not reported; ARAT: action research arm test (score range of 0-57, high score indicating normal ability); addl.: additional; SD: standard deviation; FTHUE: functional test of the hemiparetic upper extremity (score range of $0-17$, high score indicating normal ability). 
suggesting that more practice results in better poststroke upper extremity motor recovery. However, data were limited and we were unable to conclude if there is a minimum threshold of practice that will result in improved upper extremity motor function after stroke.

4.2. Strengths and Limitations. Data from the available studies are promising, but have been interpreted with caution, as there are limitations of this review. First, developing both sensitive and specific search criteria to identify appropriate articles was difficult. Ideally, search criteria will identify articles based on the population, intervention, outcomes, and methodology of interest. While a poststroke population could be identified, initial attempts to use search strategies to identify any specific types of poststroke interventions were unsuccessful, primarily because of a lack of consistency in the use of subject headings or keywords. The decision was made to use broad search terms that would provide a sensitive, albeit unspecific list of studies and this resulted in the large number of titles initially searched. This issue emphasizes the need for diligence by authors in using consistent terminology when submitting studies in this field. The search was further enhanced by hand searching bibliographies, which has been shown to be an effective supplement to electronic searches [43]. Therefore, while we believe the search is thorough, it is possible that other studies exist, especially in languages other than English.

Second, an important aspect of our questions was whether the active ingredient of task produced better outcomes than usual therapy. It was clearly evident that the definition of task is not consistent across studies, ranging from exercise to reaching activities to the practice of usual daily activities like dressing. A lack of clarity in terms of definition as well as terminology (e.g., task specific training, taskoriented training, and functional training) made it difficult to accurately identify studies of task-oriented practice. We chose to focus on studies comparing task-oriented practice as defined by Winstein and Wolf [23], which focuses on remediating activity limitations, as opposed to individual upper extremity impairments and skill development opposed to movement. Studies that described the use of another type of intervention in either the experimental or control group (e.g., NeuroDevelopmental Therapy or Constraint Induced Movement Therapy) were excluded since they were not evaluating task-oriented practice per se; however, it is possible that some element of task-oriented practice was used in these interventions. Although we suspect that this is not a likely scenario, without thorough and detailed descriptions of the interventions in each study, it is possible.

Third, while all studies scored four or above in the methodological evaluation, only one study was free from bias [40], while three studies had a lack of similarity in baseline characteristics, probably due to small sample sizes [37-39]. These baseline differences, especially in upper extremity motor function, may have made it difficult to demonstrate a statistically significant effect (and there was no indication whether statistical adjustments were made in the analysis). In addition, four of the six studies did not have complete follow-up of participants (one of the studies had less than $80 \%$ follow-up [42]). The methodological problems of small sample size and differences in baseline characteristics between groups was most likely compounded by loss to follow-up, further decreasing the likelihood finding an effect.

Fourth, not all studies identified a primary outcome measure so we chose to use the measure that theoretically should show the greatest effect, and that was administered at the end of the intervention period. This approach provided results under ideal circumstances (i.e., outcomes measured after the largest dose of the intervention and with the most sensitive tool) and could result increase the potential for Type 1 error. Of the three studies that demonstrated a statistically significant effect of practice, two had identified the ARAT as a primary outcome measure $[39,41]$ so the results of the third study by Harris et al. should be interpreted cautiously [40].

Finally, although each of the studies used deliberate task-oriented practice as the fundamental component of the intervention, there was variation in how interventions were delivered. For example, the study by Harris et al. [40] used a self-administered intervention, while the intervention in the study by Kwakkel et al. [41] was therapist administered. The lack of consistency between interventions made it inappropriate to meta-analyse study results, and therefore a minimum threshold of task-oriented practice could not be determined.

Although there are issues in terms of the design, conduct and analysis of some of the studies, it should be noted that five out of the six journals in which the studies were published have adopted the CONSORT statement for reporting clinical trials, and adherence to the statement is intended to improve the quality of reporting [44].

4.3. Clinical Perspectives. Although there are limitations to this review, it highlights two key messages; the use and definition of task varies greatly in clinical studies as well as clinical practice and that we do not know the optimal "dose" (intensity, frequency and duration) of task to improve upper extremity motor impairment. The problem of lack of clarity in defining task has been discussed previously in this review and by other authors [30]. The issue of the optimal dose remains important and unanswered. Studies on the use of task in clinical settings report that task is used in only about half of the therapy sessions [45] and for about $1 / 3$ of the length of the session [46]. It is possible that a lack of clarity in terms of "what is task" and "minimum dose" is leading therapists to underutilize task-oriented practice. Implementing a treatment regimen based on frequency and duration alone is not difficult, but the quantification and implementation of intensity remains problematic due to these uncertainties and is further complicated if therapists are uncertain of the specifics of task-oriented practice. Recent efforts to define intensity in terms of poststroke motor rehabilitation offer a definition based on the amount of work the patient performs [26]. This definition does not consider the amount of the "active ingredient" included in the intervention. For task-oriented practice, quantification of intensity has to incorporate the amount of task practice occurring. Working harder will only produce better poststroke motor recovery if the work (i.e., task-oriented practice) is aimed at producing the desired 
neurophysiologic changes and subsequent improvement in activity, and does not invoke adverse effects.

In summary, we chose to look specifically at the effect of task-oriented practice on poststroke upper limb motor recovery compared to usual care, using randomized controlled trials of patients in the subacute phase after stroke, a group that is understudied but most likely to benefit from interventions aimed at maximizing neuroplasticity [30]. The criteria for review do not seem outwardly restrictive and yet they resulted in only six studies eligible for consideration. The differences between included studies, specifically the intervention and dosage of the intervention, made it inappropriate to further combine these data in any statistical manner. These considerations highlight the need for research that (a) clearly defines the intervention, (b) clearly defines the intensity, frequency, and duration of the intervention (dosage), and (c) uses similar outcome measures.

While the results of this review are inconclusive, they do provide evidence of a possible effect of task-oriented practice, although a specific amount required could not be quantified. These conclusions are consistent with Canadian and international guidelines that either provide minimum amounts of therapy required, with little evidence in terms of effect, or call for increased intensity in therapy without further clarification or quantification of the meaning of increased or intensity $[47,48]$. With limited rehabilitation resources, governments need to support well-designed clinical trials that address the question of intensity, frequency, and duration of task-oriented practice. Alternative solutions for enabling additional practice must be considered such as selfadministered task-oriented practice as was shown effective by Harris and colleagues [40]. Standardized protocols, along with studies designed and powered to examine the dose response, will provide the needed information in terms of the effect and the optimal dosage threshold.

\section{Conclusions}

There are few studies that have looked solely at the effectiveness of task-oriented practice on poststroke upper extremity motor recovery in the subacute phase. The available evidence indicates that task-oriented practice may result in improved motor recovery and may ultimately result in improvements in poststroke activity. Large, well designed, and conducted randomized controlled trials, using standardized protocols with consistent definitions, that examine the response to various doses of task-oriented practice, are required to confirm the effect of task-oriented practice and to determine a minimum threshold of practice at which motor recovery occurs.

\section{Conflict of Interests}

The authors declared they have no conflict of interest.

\section{Acknowledgment}

The authors would like to thank Shannon Buckley for her help and insights to identify appropriate search terms and her assistance in performing the searches.

\section{References}

[1] S. C. Johnston, S. Mendis, and C. D. Mathers, "Global variation in stroke burden and mortality: estimates from monitoring, surveillance, and modelling," The Lancet Neurology, vol. 8, no. 4, pp. 345-354, 2009.

[2] K.-S. Hong and J. L. Saver, "Quantifying the value of stroke disability outcomes: WHO global burden of disease project disability weights for each level of the modified rankin scale," Stroke, vol. 40, no. 12, pp. 3828-3833, 2009.

[3] P. Langhorne, F. Coupar, and A. Pollock, "Motor recovery after stroke: a systematic review," The Lancet Neurology, vol. 8, no. 8, pp. 741-754, 2009.

[4] F. Ferrarello, M. Baccini, L. A. Rinaldi et al., "Efficacy of physiotherapy interventions late after stroke: a meta-analysis," Journal of Neurology, Neurosurgery and Psychiatry, vol. 82, no. 2, pp. 136-143, 2011.

[5] E. V. Cooke, K. Mares, A. Clark, R. C. Tallis, and V. M. Pomeroy, "The effects of increased dose of exercise-based therapies to enhance motor recovery after stroke: a systematic review and meta-analysis," BMC Medicine, vol. 8, article 60, 2010.

[6] B. French, L. Thomas, M. Leathley et al., "Does repetitive task training improve functional activity after stroke? A Cochrane systematic review and meta-analysis," Journal of Rehabilitation Medicine, vol. 42, no. 1, pp. 9-14, 2010.

[7] C. P. Latimer, J. Keeling, B. Lin, M. Henderson, and L. A. Hale, "The impact of bilateral therapy on upper limb function after chronic stroke: a systematic review," Disability and Rehabilitation, vol. 32, no. 15, pp. 1221-1231, 2010.

[8] S. H. Peurala, M. P. Kantanen, T. Sjögren, J. Paltamaa, M. Karhula, and A. Heinonen, "Effectiveness of constraint-induced movement therapy on activity and participation after stroke: a systematic review and meta-analysis of randomized controlled trials," Clinical Rehabilitation, vol. 26, no. 3, pp. 209-223, 2012.

[9] K. E. Laver, S. George, S. Thomas, J. E. Deutsch, and M. Crotty, "Virtual reality for stroke rehabilitation," Cochrane Database of Systematic Reviews, vol. 9, Article ID CD008349, 2011.

[10] K. Hayward, R. Barker, and S. Brauer, "Interventions to promote upper limb recovery in stroke survivors with severe paresis: a systematic review," Disability and Rehabilitation, vol. 32, no. 24, pp. 1973-1986, 2010.

[11] A. A. A. Timmermans, A. I. F. Spooren, H. Kingma, and H. A. M. Seelen, "Influence of task-oriented training content on skilled arm-hand performance in stroke: a systematic review," Neurorehabilitation and Neural Repair, vol. 24, no. 9, pp. 858870, 2010.

[12] J. M. Veerbeek, M. Koolstra, J. C. F. Ket, E. E. H. van Wegen, and G. Kwakkel, "Effects of augmented exercise therapy on outcome of gait and gait-related activities in the first 6 months after stroke: a meta-analysis," Stroke, vol. 42, no. 11, pp. 3311-3315, 2011.

[13] L. Oujamaa, I. Relave, J. Froger, D. Mottet, and J.-Y. Pelissier, "Rehabilitation of arm function after stroke. Literature review," Annals of Physical and Rehabilitation Medicine, vol. 52, no. 3, pp. 269-293, 2009.

[14] A. Zimmermann-Schlatter, C. Schuster, M. A. Puhan, E. Siekierka, and J. Steurer, "Efficacy of motor imagery in poststroke rehabilitation: a systematic review," Journal of NeuroEngineering and Rehabilitation, vol. 5, article 8, 2008. 
[15] D. Bonaiuti, L. Rebasti, and P. Sioli, "The constraint induced movement therapy: a systematic review of randomised controlled trials on the adult stroke patients," Europa Medicophysica, vol. 43, no. 2, pp. 139-146, 2007.

[16] A. Henderson, N. Korner-Bitensky, and M. Levin, "Virtual reality in stroke rehabilitation: a systematic review of its effectiveness for upper limb motor recovery," Topics in Stroke Rehabilitation, vol. 14, no. 2, pp. 52-61, 2007.

[17] M. L. Urton, M. Kohia, J. Davis, and M. R. Neill, "Systematic literature review of treatment interventions for upper extremity hemiparesis following stroke," Occupational Therapy International, vol. 14, no. 1, pp. 11-27, 2007.

[18] G. Kwakkel, R. van Peppen, R. C. Wagenaar et al., "Effects of augmented exercise therapy time after stroke: a meta-analysis," Stroke, vol. 35, no. 11, pp. 2529-2539, 2004.

[19] R. N. Barker and S. G. Brauer, "Upper limb recovery after stroke: the stroke survivors' perspective," Disability and Rehabilitation, vol. 27, no. 20, pp. 1213-1223, 2005.

[20] P. Craig, P. Dieppe, S. Macintyre, S. Michie, I. Nazareth, and M. Petticrew, "Developing and evaluating complex interventions: the new Medical Research Council guidance," BMJ, vol. 337, article a1655, 2008.

[21] P. Santaguida, M. Oremus, K. Walker, L. R. Wishart, K. L. Siegel, and P. Raina, "Systematic reviews identify important methodological flaws in stroke rehabilitation therapy primary studies: review of reviews," Journal of Clinical Epidemiology, vol. 65, no. 4, pp. 358-367, 2012.

[22] K. N. Arya, R. Verma, R. K. Garg, V. P. Sharma, M. Agarwal, and G. G. Aggarwal, "Meaningful task-specific training (MTST) for stroke rehabilitation: a randomized controlled trial," Topics in Stroke Rehabilitation, vol. 19, no. 3, pp. 193-211, 2012.

[23] C. J. Winstein and S. L. Wolf, "Task-oriented training to promote upper extremity recover," in Stroke Recovery and Rehabilitation, J. Stein, R. L. Harvery, R. F. Macko, C. J. Winstein, and R. D. Zorowitz, Eds., pp. 267-290, Demos Medical, New York, NY, USA, 2009.

[24] R. A. Schmidt and T. D. Lee, "Conditions of practice," in Motor Control and Learning: A Behavioral Emphasis, pp. 347-392, Human Kinetics, Ontario, Canada, 2011.

[25] G. Kwakkel, R. C. Wagenaar, T. W. Koelman, G. J. Lankhorst, and J. C. Koetsier, "Effects of intensity of rehabilitation after stroke: a research synthesis," Stroke, vol. 28, no. 8, pp. 1550-1556, 1997.

[26] S. J. Page, A. Schmid, and J. E. Harris, "Optimizing terminology for stroke motor rehabilitation: recommendations from the American Congress of Rehabilitation Medicine Stroke Movement Interventions Subcommittee," Archives of Physical Medicine and Rehabilitation, vol. 93, no. 8, pp. 1395-1359, 2012.

[27] G. Kwakkel, "Impact of intensity of practice after stroke: issues for consideration," Disability and Rehabilitation, vol. 28, no. 1314, pp. 823-830, 2006.

[28] A. C. Wallace, P. Talelli, M. Dileone et al., "Standardizing the intensity of upper limb treatment in rehabilitation medicine," Clinical Rehabilitation, vol. 24, no. 5, pp. 471-478, 2010.

[29] C. J. Winstein, S. L. Wolf, A. W. Dromerick et al., "Interdisciplinary Comprehensive Arm Rehabilitation Evaluation (ICARE): a randomized controlled trial protocol," $\mathrm{BMC} \mathrm{Neu-}$ rology, vol. 13, no. 1, article 5, 2013.

[30] M. G. Bowden, M. L. Woodbury, and P. W. Duncan, "Promoting neuroplasticity and recovery after stroke: future directions for rehabilitation clinical trials," Current Opinion in Neurology, vol. 26, no. 1, pp. 37-42, 2013.
[31] P. Langhorne, J. Bernhardt, and G. Kwakkel, "Stroke rehabilitation," The Lancet, vol. 377, no. 9778, pp. 1693-1702, 2011.

[32] R. L. Birkenmeier, E. M. Prager, and C. E. Lang, "Translating animal doses of task-specific training to people with chronic stroke in 1-hour therapy sessions: a proof-of-concept study," Neurorehabilitation and Neural Repair, vol. 24, no. 7, pp. 620 $635,2010$.

[33] S. Sehatzadeh, "Effectiveness of increased intensity of rehabilitation in post-stroke patients: a rapid review," Tech. Rep., Toronton, Canada, 2013.

[34] World Health Organization, Towards a Common Language for Functioning, Disability and Health: ICF, World Health Organization, Geneva, Switzerland, 2002.

[35] World Health Organization, "International Classification of Functioning, Disability and Health," 2001, http://www.who.int/ classifications/icf/site/intros/ICF-Eng-Intro.pdf.

[36] A. Liberati, D. G. Altman, J. Tetzlaff et al., "The PRISMA statement for reporting systematic reviews and meta-analyses of studies that evaluate healthcare interventions: explanation and elaboration," BMJ, vol. 339, article b2700, 2009.

[37] J. Blennerhassett and W. Dite, "Additional task-related practice improves mobility and upper limb function early after stroke: a randomised controlled trial," Australian Journal of Physiotherapy, vol. 50, no. 4, pp. 219-224, 2004.

[38] C. Donaldson, R. Tallis, S. Miller, A. Sunderland, R. Lemon, and V. Pomeroy, "Effects of conventional physical therapy and functional strength training on upper limb motor recovery after stroke: a randomized phase II study," Neurorehabilitation and Neural Repair, vol. 23, no. 4, pp. 389-397, 2009.

[39] C. Han, Q. Wang, P. P. Meng, and M. Z. Qi, "Effects of intensity of arm training on hemiplegic upper extremity motor recovery in stroke patients: a randomized controlled trial," Clinical Rehabilitation, vol. 27, no. 1, pp. 75-81, 2013.

[40] J. E. Harris, J. J. Eng, W. C. Miller, and A. S. Dawson, "A selfadministered graded repetitive arm supplementary program (GRASP) improves arm function during inpatient stroke rehabilitation: a multi-site randomized controlled trial," Stroke, vol. 40, no. 6, pp. 2123-2128, 2009.

[41] G. Kwakkel, R. C. Wagenaar, J. W. R. Twisk, G. J. Lankhorst, and J. C. Koetsier, "Intensity of leg and arm training after primary middle-cerebral-artery stroke: a randomised trial," The Lancet, vol. 354, no. 9174, pp. 191-196, 1999.

[42] C. J. Winstein, D. K. Rose, S. M. Tan, R. Lewthwaite, H. C. Chui, and S. P. Azen, "A randomized controlled comparison of upper-extremity rehabilitation strategies in acute stroke: a pilot study of immediate and long-term outcomes," Archives of Physical Medicine and Rehabilitation, vol. 85, no. 4, pp. 620-628, 2004.

[43] S. Hopewell, M. Clarke, C. Lefebvre, and R. Scherer, "Handsearching versus electronic searching to identify reports of randomized trials," Cochrane Database of Systematic Reviews, no. 2, Article ID MR000001, 2007.

[44] S. Ghimire, E. Kyung, W. Kang, and E. Kim, "Assessment of adherence to the CONSORT statement for quality of reports on randomized controlled trial abstracts from four high-impact general medical journals," Trials, vol. 13, article 77, 2012.

[45] C. E. Lang, J. R. MacDonald, D. S. Reisman et al., "Observation of amounts of movement practice provided during stroke rehabilitation," Archives of Physical Medicine and Rehabilitation, vol. 90, no. 10, pp. 1692-1698, 2009. 
[46] L. De Wit, H. Kamsteegt, B. Yadav, G. Verheyden, H. Feys, and W. De Weerdt, "Defining the content of individual physiotherapy and occupational therapy sessions for stroke patients in an inpatient rehabilitation setting. Development, validation and inter-rater reliability of a scoring list," Clinical Rehabilitation, vol. 21, no. 5, pp. 450-459, 2007.

[47] M. P. Lindsay, G. Gubitz, M. Bayley et al., "Canadian best practice recommendations for stroke care (update 2010)," Tech. Rep., Ontario, Canada, 2010.

[48] N. Foley, S. Pereira, K. Salter, M. Meyer, J. A. McClure, and R. Teasell, "Are recommendations regarding inpatient therapy intensity following acute stroke really evidence-based?" Topics in Stroke Rehabilitation, vol. 19, no. 2, pp. 96-103, 2012. 


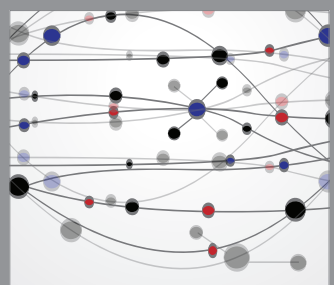

The Scientific World Journal
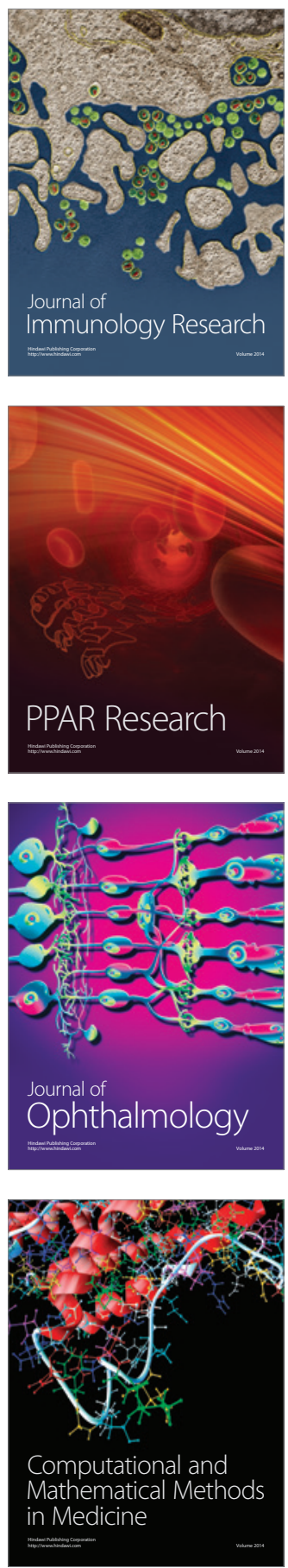

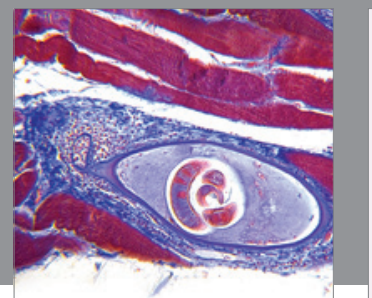

Gastroenterology

Research and Practice
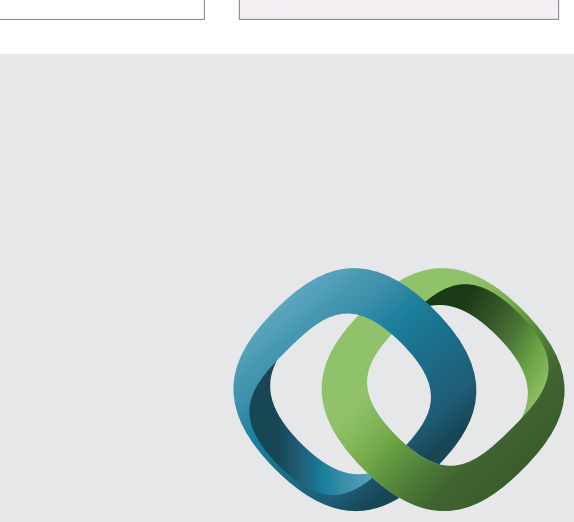

\section{Hindawi}

Submit your manuscripts at

http://www.hindawi.com
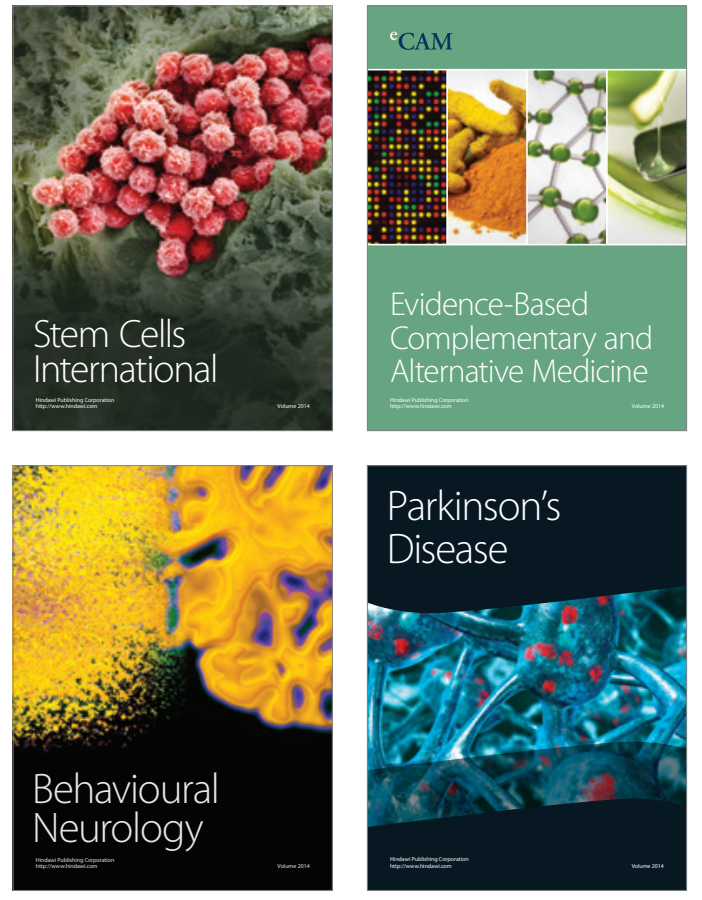
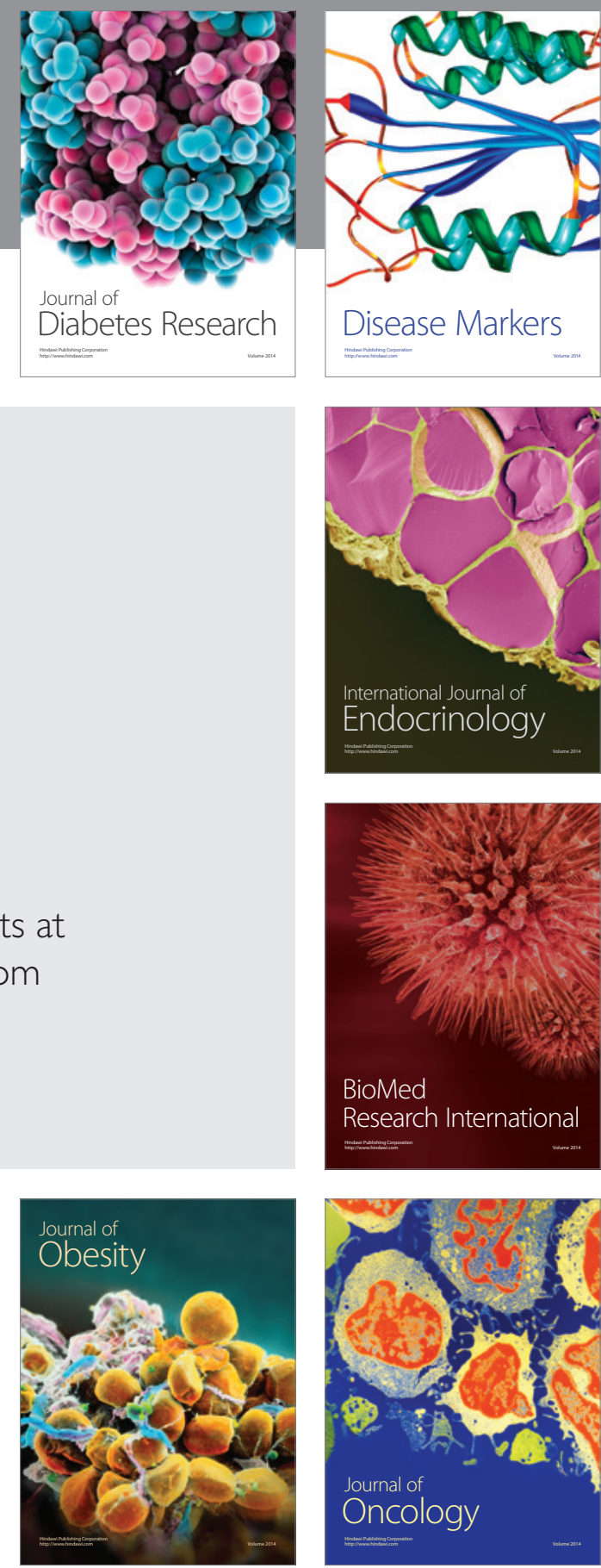

Disease Markers
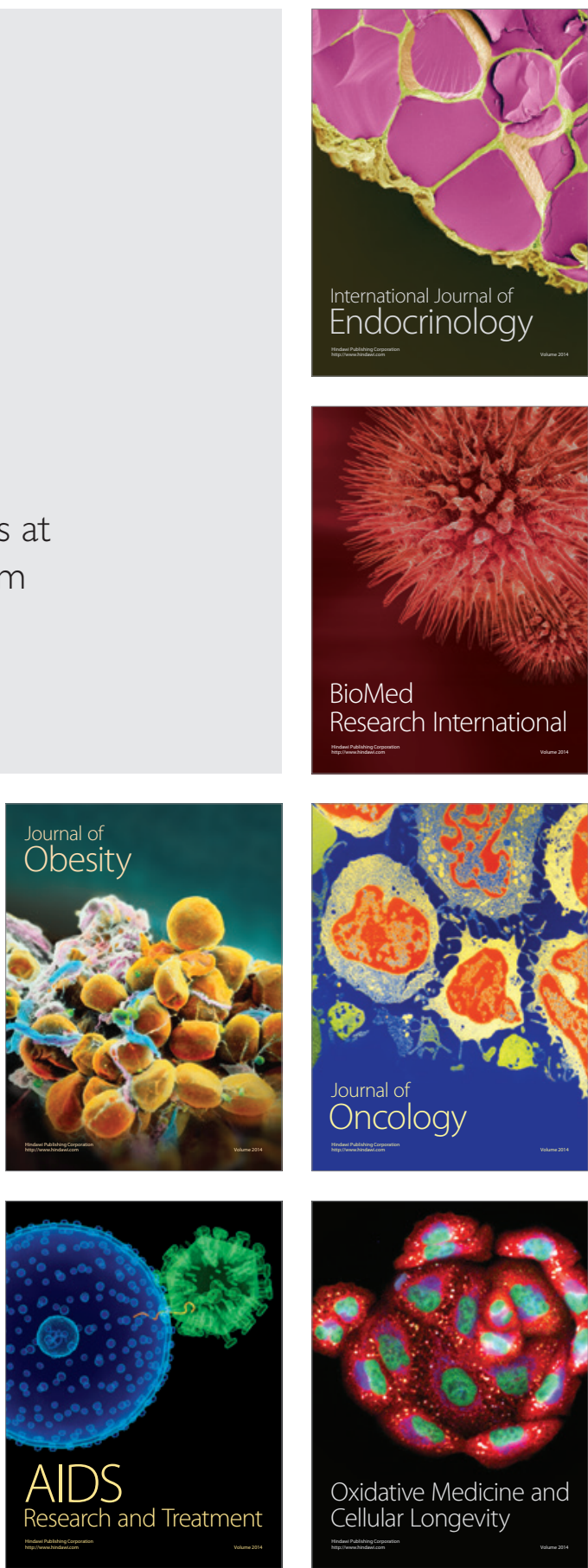